\title{
Spatial distribution of economic activities in heritage cities: The case of the historic city of Toledo, Spain
}

\author{
Borja Ruiz-Apilánez ${ }^{1}$, Eloy Solís ${ }^{1}$, Irene García-Camacha ${ }^{1}$, Vicente \\ Romero de Avila ${ }^{2}$, Carmen Alía ${ }^{1}$, Raúl Martín ${ }^{1}$ \\ ${ }^{1}$ Escuela de Arquitectura, Universidad de Castilla-La Mancha, Toledo, Spain \\ ${ }^{2}$ Escuela de Ingenieros de Caminos, Universidad de Castilla-La Mancha, Ciudad Real, Spain \\ E-mail: borja.ruizapilanez@uclm.es, eloy.solis@uclm.es, vicente.romeroavila@uclm.es, irene. \\ garciacamacha@uclm.es, carmen.alia@alu.uclm.es,raul.mmartin@uclm.es
}

\begin{abstract}
This paper presents the initial results of an ongoing research on the spatial distribution of economic activities in Spanish heritage cities. The study investigates the influence of spatial accessibility and proximity to monuments on the location of businesses in the historic city of Toledo. The research includes two surveys of the entrances to monuments and ground floor businesses within the old town, and uses space syntax techniques and tools to determine spatial accessibility. Then, a GIS model of the street segments of the city registers this information. Statistical analysis suggests spatial accessibility and proximity to monuments have a significant impact on the distribution of business in the old town and investigates further to understand and quantify this effect. However, it does not find strong correlations between the considered variables nor a suitable model to explain the differences in business intensity across the street segments within the historic city of Toledo.
\end{abstract}

Keywords: urban morphology, urban economics, space syntax, land uses, heritage cities

Introduction, research objective and case study

Cities are socio-physical entities on a continuous and continuing evolution. For the case of historic cities in Spain - also in Europe and many other world regions - this process of readjustment has been going on now for over two thousand years. After this time, far from reaching any sort of equilibrium, many old towns suffer serious social and material decline. Among these ancient human settlements, our society recognises heritage cities are the most valuable fragments of built environment. As such, they deserve our greatest efforts to assure they survive as urban tissues in the best shape. To guarantee their endurance and sustainability it is important to lookout their socio-economic status even better than their monuments.

To date, existing studies on the economy in historic cities have been formulated without invocating space. It has been widely considered that space is a background to human activity. Quite differently, space syntax approach considers space "as an intrinsic aspect of everything human beings do" (Hillier \& Vaughan, 2007: 208).

Previous research claim that the spatial configuration of the street layout has an important effect on pedestrian movement (Hillier, 2002; Hillier et al., 1993), the location of prominent urban elements (Hanson, 1989; Karimi, 2000), and the distribution of land uses types and densities (Froy, 2015; Kim \& Sohn, 2002; Lee \& Kim, 2009; Min, Moon, \& Kim, 2007; Narvaez, Penn, \& Griffiths, 2013; Shen \& Karimi, 2017).

This research investigates the spatial logic of economic activities in heritage cities with high tourism activity, using space syntax theories and tools. The main objective is to understand the distribution of ground floor businesses, or, 
in Conzen's words, their "pattern of urban land use" (Conzen, 1960: 4).

Based on previous research and observations (Troitiño et al., 1998; Zárate, 2007; RuizApilánez et al., 2015), the hypothesis is that high spatial accessibility and proximity to monuments might be important to understand the location of economic activities in heritage cities, such as the historic city of Toledo.

Toledo is the capital of Castille-La Mancha and was the imperial capital during the 16th century. Currently, it has a population of 83,000 people. 11,000 inhabitants live in the old town. The study focuses inside the walls of the city, covering an area close to one square kilometre. The old town has an organic medieval street network. The river Tagus surrounds it on two thirds of its perimeter and it has limited connections with the rest of the city, which has been mostly developed since the mid-20th century. It shows a great number of monuments - including buildings such as the cathedral, the town hall, and university buildings, as well as other constructions such as walls, gates, and bridges - and, since the late 1990s motor traffic is restricted in most of the street network.

\section{Research methods}

This section addresses two main issues. First, how this study contemplates the three investigated aspects of the urban tissue within the historic city of Toledo: (1) the distribution of businesses located on the ground floor, (2) the spatial accessibility of streets, and (3) the location of monuments. Second, how this study analyses the relation among these three factors.

In the first place, the method determines a set of variables that represent the three investigated aspects. The following paragraphs describe these variables in detail but, before, it is important to explain the method needs to define a spatial element to which all variables are linked. This spatial element is the street segment. In this research, street segments are generated after an axial map, using Depthmap software. The axial map comprises the whole city of Toledo, being the old town a part of it. The street segment model (Figure 1) is an updated version of an earlier one that was tested in previous research, e.g. Arnaiz et al. (2013) and Ruiz-Apilánez et al. (2015). Since motor traffic is restricted in the old townmost streets are only accessible to neighbours

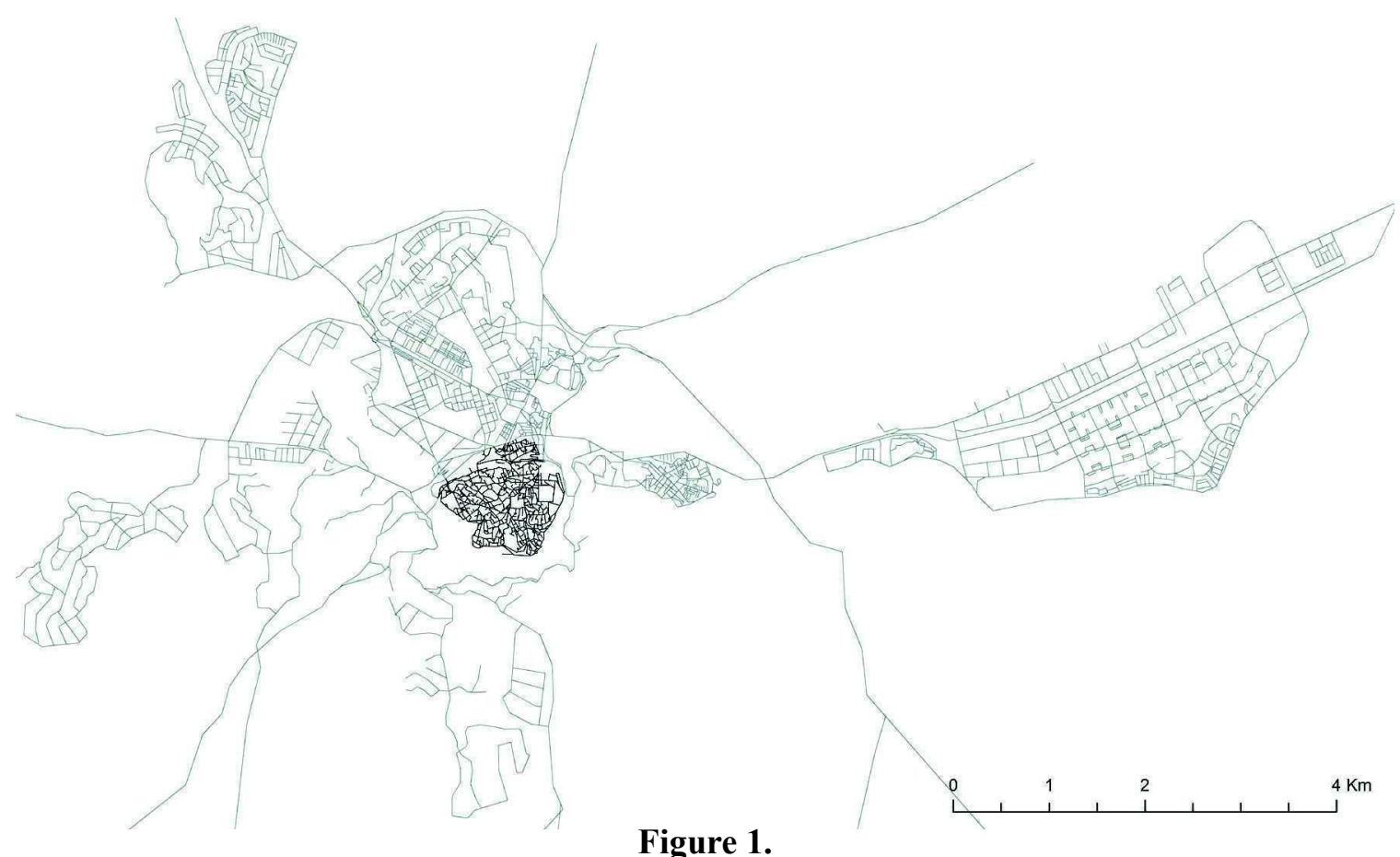

Street segment model of Toledo. The historic city (in black) surrounded by the rest of the city (in grey) 

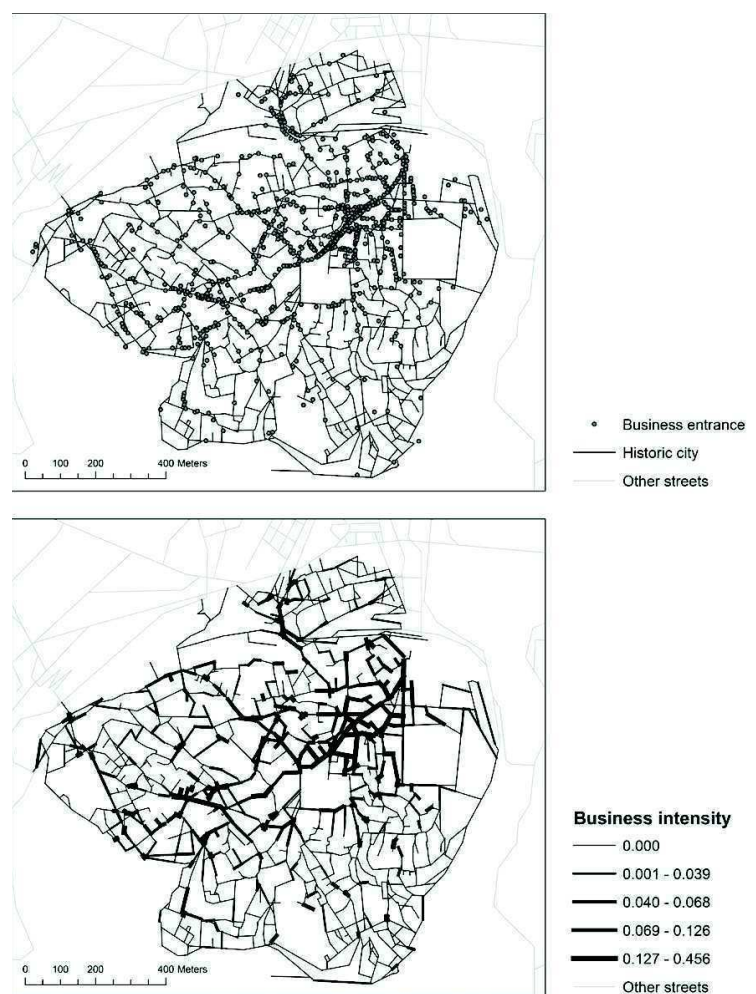

Figure 2.

Ground floor business in the historic city of Toledo. Above: location of business entrances. Below: business intensity (number of business entrances per metre of street segment)

and deliveries - movement within the historic city is mainly on foot. Consequently, the current model includes all the streets and accesses reserved for pedestrians, including two escalators in the north and northeast edges of the historic city. The method uses GIS to register the information regarding the three variables by linking them to the street segments.

This study deals with the location of businesses through the variable 'business intensity'. To calculate this variable, the first necessary step is to register the location of the ground floor accesses to these businesses (Figure 2, above). Then, each entrance is assigned to the adjacent street segment. Most entrances are linked to only one street segment, but there are a few exceptions, such as entrances on street corners, that are assigned to two street segments. As a result, each segment has a number of business doors linked to it.
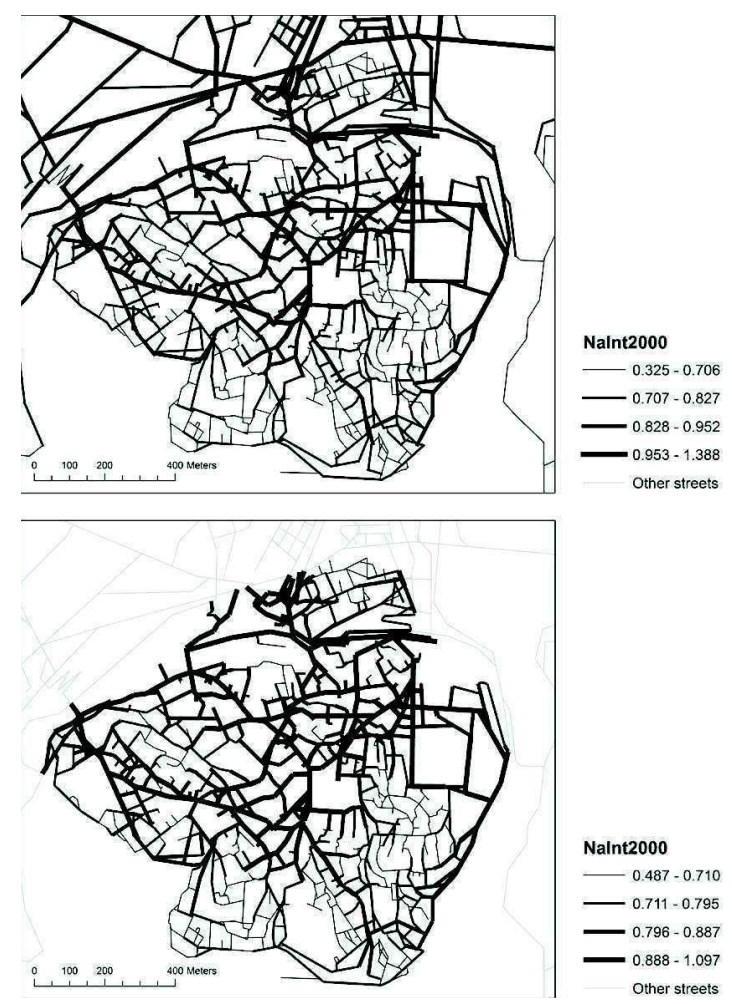

Figure 3.

Spatial accessibility in the historic city of Toledo. Above: space syntax variables are obtained considering the whole city. Normalised integration ( $r$ $=2000 \mathrm{~m}$ ). Below: statistical analysis only considers the street segments in the historic city. Normalised integration $(r=2000 \mathrm{~m})$ for the street segments in the historic city

Businesses intensity is the result of dividing the number of entrances between the length of the corresponding street segment. This variable mostly represents the retail intensity of each street segment (Figure 2, below).

To consider the position of these entrances within the street network, the street segment model is analysed using space syntax techniques and tools. The method assigns a set of spatial variables to each street segment, including normalised integration and normalised choice (for radii 500, 1000, and 2000 metres, and radius $\mathrm{n}$, which considers each street segment in relation to the full model). This series of variables represent the spatial accessibility of each street segment. Integration and choice are the two fundamental space syntax measures of "accessibility for to-movement" and "through-movement potential" (Hillier \& Vaughan, 2007: 212). The calculations of the space syntax variables consider the full model 


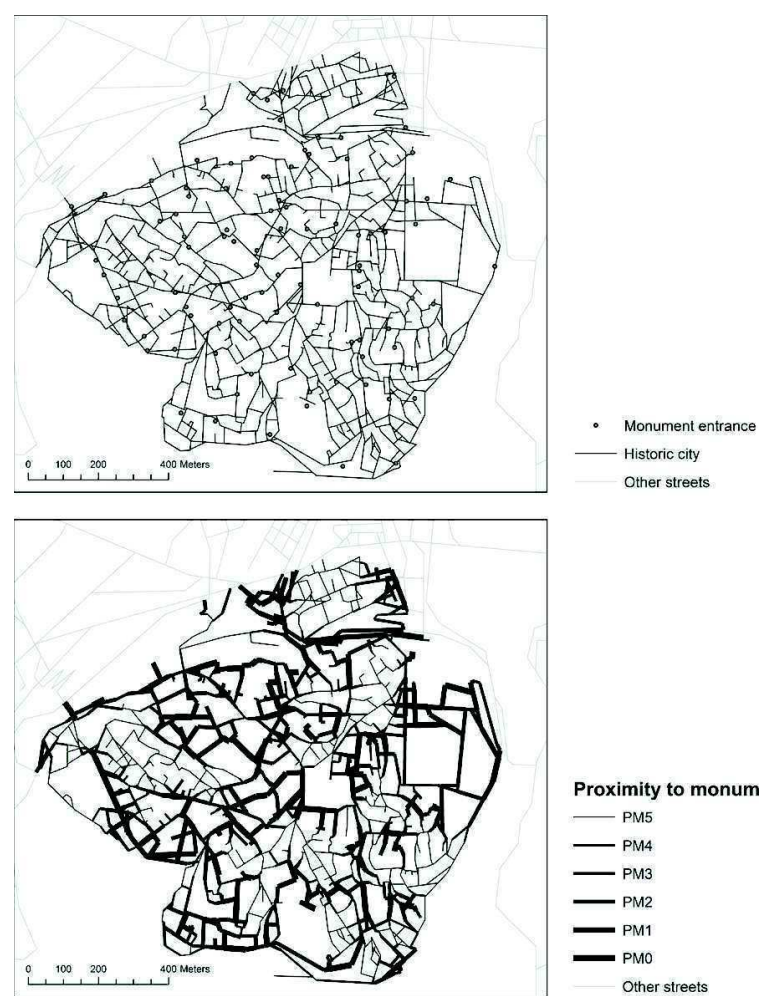

Figure 4.

Presence of and proximity to monuments in the historic city of Toledo. Above: location of monument entrances. Below: proximity to monuments.

although the statistical analysis only considers the segments within the historic city (Figure 3).

There, statistical analysis will show moderate-high correlation between the eight space syntax variables, being normalise integration $(\mathrm{r}=2000 \mathrm{~m})$ the space syntax variable that better explains the location of economic activity. Therefore, in some phases of the analysis, the spatial accessibility of the street segments is characterised only by this one variable. The following section further describes this matter.

To study the role of monuments, the method defines the variable 'proximity to monuments', which provides a measure of how distant a street segment is to a monument entrance. To determine the variable it is first necessary to register all entrances to listed monuments in the historic city (Figure 4, above). The study includes all listed monuments in the old cityat national, regional and local levels. This factor (categorical) variable classifies the street segments in six groups, from PM0 to PM5 (Figure 4, below).

Street segments with an entrance to a monument belong to group PM0. Street segments connected to any of the preceding segments (PM0) fall into group PM1. Street segments linked to a segment of the preceding group (PM1) but not in the first group (PM0) belong to group PM2. Correspondingly, the method determines the street segments that go to groups PM3 and PM4. Street segments not included in any of the five preceding groups (PM0 to PM4) constitute group PM5.

Thus, this variable indicates if a street segment either gives access to a monument (PM0), is one to four segments away from a monument entrance (PM1 to PM4), or is more than four segments away from a monument entrance (PM5).

Once the three aspects are characterised by the three kinds of variables - economic (businesses intensity), spatial accessibility (space syntax variable/s), and historic/touristic (proximity to monuments) - the method applies further statistical analysis to investigate the relation between these variables.

The first two parts of the study, which deal with the location of businesses, include two complementary analyses. The first one studies the corresponding variable as dichotomous, displaying only two possible values: zero or one. Zero means the street segment has no access to businesses on the ground floor; one means the street segment has one or more entrances to businesses. The second investigates the continuous numeric variable 'business intensity'-number of entrances to ground floor businesses, considering both sides of a given street segment, divided between the length of the segment. In the historic city of Toledo, when different to zero, this variable shows positive values from 0.003 (approximately, one entrance in 300 metres) to 0.456 (approximately, one door every 2 metres, or 45 entrances in 100 metres).

Each of the three following sections discusses the results of analysing the relation between: (1) location of businesses and spatial accessibility; (2) location of businesses and location of/proximity to monuments; and (3) location of/proximity to monuments and spatial accessibility. 

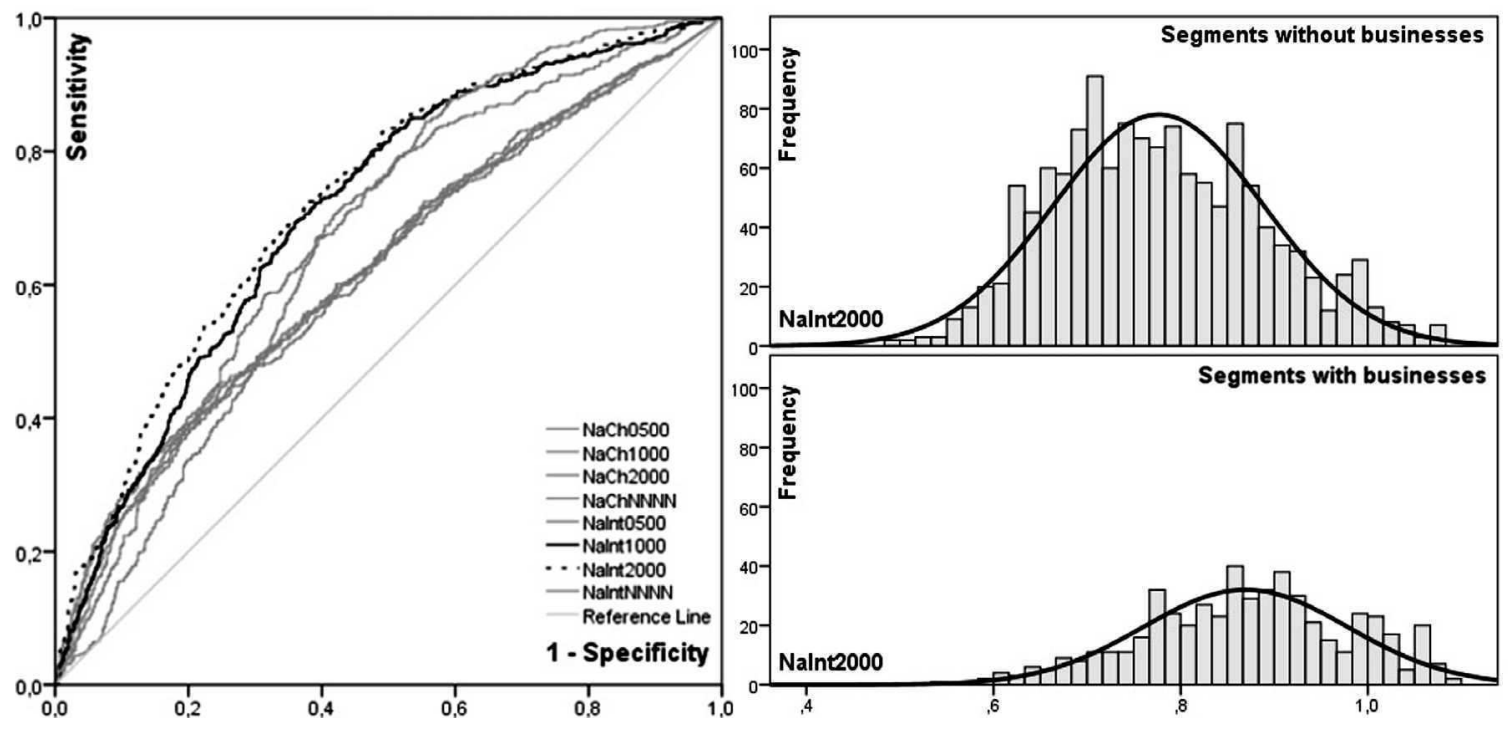

Figure 5.

Left: ROC curves for businesses prediction using space syntax variables as indicator (normalised integration and choice for radii 500, 1000, and 2000 metres, and radius $n$ ). Right: histograms and probability density functions of spatial accessibility (normalised integration, $r=2000 \mathrm{~m}$ ) for street segments with (below) and without (above) businesses.

\section{Location of businesses and spatial accessibility}

This section investigates if the spatial accessibility of a given street segment affects the presence and number of businesses on the ground floor of the adjacent buildings.

In the first place, the study differentiates between two groups of street segments within the historic city: street segments with entrances to businesses and street segments without them. Then, statistical analysis explores if there are significant differences in accessibility between these two groups. The study considers a set of eight space syntax variables: normalised integration and normalised choice for radii 500,1000 , and 2000 metres, and radius $n$ (each segment in relation to every other segment of the model).

Two-sample $t$ test examine whether or not two independent populations - segments with and without businesses, respectively, 527 and 1321 segments in the old city - have different mean values on these space syntax variables. The results show that the differences are significant for the eight investigated variables ( $p<0.05$ in all cases), with higher means for the streets segments with businesses.

Then, considering each of the space syntax variables to be the independent variable, statistical analysis investigates if any of these spatial variables can discriminate between the two kinds of street segments (with vs. without business entrances). For this purpose, the study generates receiver operating characteristic (ROC) curves (Figure 5, left).

The area under the curve (AUC) indicates the probability that the given independent variable - the one used to plot the curveproperly classifies the street segment as a segment with/without entrances to businesses. The test accepts probabilities higher than 0.7. This is the case of integration, $r=1000 \mathrm{~m}$ (AUC $=0.709$ ), and integration, $r=2000 \mathrm{~m}$ (AUC $=0.725$ ). The latter is the spatial variable that better discriminates between the two kinds of street segments.

For example, setting a cut-off value of 0.79 for normalised integration $(r=2000 \mathrm{~m})$, sensitivity and specificity values are 0.75 and 0.60 , respectively. In this context, sensitivity is the ability of this spatial variable to correctly classify a street segment as one with business entrances; while specificity is the ability of this spatial variable to correctly classify a segment as one without business entrances (Figure 5, right).

Once the analysis has shown there is an effect of the spatial variables on the economic variable, in the sense that some of the former 
can discriminate between street segments with businesses and without them, the study intends to understand this influence between variables better.

To address this question, the next step was to evaluate possible correlations between business intensity and the defined set of space syntax variables. However, Spearman coefficient (SC) values show weak correlation between business intensity and six accessibility variables $(\mathrm{SC}<0.3)$, and weak-to-moderate correlation between business intensity and the other two: integration, $\mathrm{r}=1000 \mathrm{~m}(\mathrm{SC}=0.34)$, and integration, $\mathrm{r}=2000 \mathrm{~m}(\mathrm{SC}=0.37)$.

There are two other remarkable facts regarding accessibility variables in the old town. First, there are high correlations $(\mathrm{SC}>$ $0.7)$ among integration variables $(0.739<\mathrm{SC}$ $<0.937)$ and choice variables $(0.895<\mathrm{SC}$ $<0.998$ ). Second, given the same radius of analysis, integration and choice variables for the same radius of analysis $(500,1000$, and $5000 \mathrm{~m}$, and $\mathrm{n})$ moderate correlations $(0.529<$ $\mathrm{SC}<0.600)$.

Since the analysis rejects any linear regression model, the study investigates other possible models. The search begun by plotting the scatter graphs for business intensity vs. each one of the space syntax variables considered in the analysis: normalised integration and choice, for radii 500, 1000, and 2000 metres, and radius $n$ (Figure 6). However, no one shows any recognizable pattern. This fact suggests that spatial variables are not enough to explain the location of business in the historic city of Toledo.

Given the impossibility of finding a model based on the considered space syntax variables, but knowing that integration, $r=1000 \mathrm{~m}$ and $\mathrm{r}=$ $2000 \mathrm{~m}$, discriminate between street segments with businesses and street segments without them, the study further investigates the relation between business intensity and integration, $r$ $=2000 \mathrm{~m}$, since it is the spatial variable that better explains the location of businesses in the historic city of Toledo.

Further statistical analysis recodes normalised integration, $\mathrm{r}=2000 \mathrm{~m}$, as a factor (categorical) variable. The recoding assigns each street segment to one of four groups (IntQ1, IntQ2, IntQ3 or IntQ4) depending on the
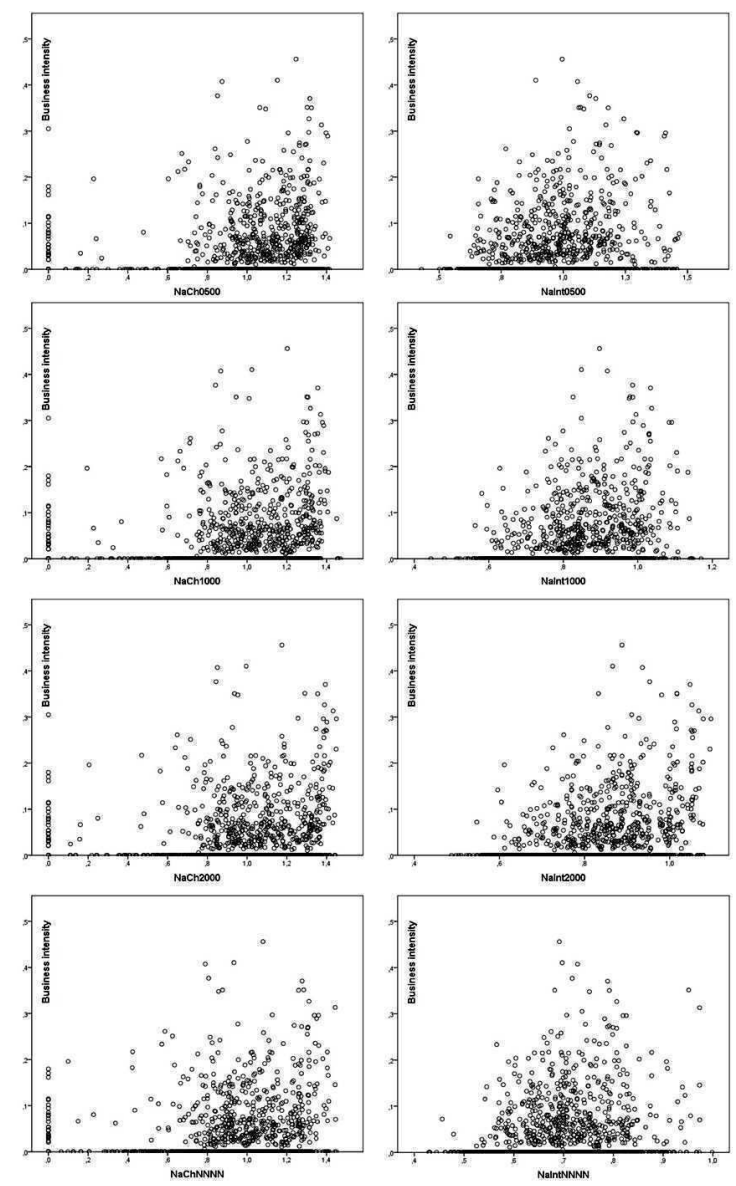

Figure 6.

Scatter graphs for business intensity vs. the eight space syntax variables considered in the study (normalised integration and choice for radii 500, 1000, and 2000 metres, and radius $n$ ).

quartile it belongs according to its integration value. IntQ1 corresponds with the first quartile (street segments with lower integration values) and IntQ4 corresponds with the fourth quartile (segments with higher integration values).

Then, the study searches throughout an ANOVA analysis a model that explains business intensity based on the quartile of the street segments. Levene test indicates the variances of the four groups are significantly different (L $=153.5 ; \mathrm{p}<0.05)$. Welch $(\mathrm{W}=68.5 ; \mathrm{p}<0.05)$ and Brown-Forsythe $(\mathrm{B}-\mathrm{F}=1112.6 ; \mathrm{p}<0.05)$ tests show there are significant differences among the mean values of the four quartiles. The predicted business intensity values for a street segment belonging to groups IntQ1, IntQ2, IntQ3, and IntQ4 are 0.006, 0.015, 0.027 , and 0.058 , respectively.

Last, Games-Howell test indicates there are significant differences $(\mathrm{p}<0.05$ in all cases) 
between the mean values of business intensity for every pair of groups (IntQ1 vs. IntQ2, IntQ1 vs. IntQ3, IntQ1 vs. IntQ4, IntQ2 vs. IntQ3, IntQ2 vs. IntQ4, and IntQ3 vs. IntQ4).

Since all the means are significantly different from each other, it is possible to state that business intensity is actually different among these four groups of street segments. In fact, the actual business intensity of the segments in the top quartile, IntQ4, suggest that the most integrated street segments (the top twenty-five per cent) do support some significant business activity. The observed mean business intensity in those segments ( 0.058 entrances per metre) corresponds with almost six business entrances in a hundred metres.

\section{Location of businesses and proximity to monuments}

The section on research methods has already presented how this study divides the street segments of the historic city in six groups based on their proximity to monuments, ranging from PM0 to PM5. Group PM0 include every street segment with direct access to a monument; segments in groups PM1 to PM4 are one to four segments away from a monument entrance, respectively; and those in PM5 are further away from any monument.

As in the preceding section, the statistical analysis that investigates if the presence of or proximity to monuments influence the location of businesses begins considering the latter as a dichotomous variable that only classifies street segments in two kinds: those with one or more business entrances and those with none. Then, the study examines the percentage of segments with and without accesses to businesses for the six groups of street segments (PM0 to PM5) depending on their proximity to a monument entrance.

Cramer's V test $(p<0.05)$ indicates there is association between the two investigated variables. Moreover, the percentage of street segments with business entrance gradually decreases from group PM0 (62.2\%) to PM5 $(19.1 \%)$. The percentages for groups PM1 to PM4 are 4.9, 31.1, 28.2, and 27.9, respectively. The analysis confirms that, in the historic city of Toledo, the number of businesses in a given street segment depends on its proximity to a monument entrance.

Second, the study investigates if it is possible to explain this relation better using an ANOVA model. To do so, economic activity is represented by the variable 'business intensity' - a continuous numeric variable defined as the number of entrances to businesses on both sides of a given street segment, divided between its length.

Levene test shows the variances of the six groups are significantly different $(\mathrm{L}=3.461$; $\mathrm{p}=0.004)$. Welch $(\mathrm{W}=2.993 ; \mathrm{p}=0.011)$ and Brown-Forsythe (B-F $=2.621 ; \mathrm{p}=0.023)$ tests show there are significant differences between the business intensity medians of the different groups, depending on their proximity to monuments. The predicted business intensity values for a street segment belonging to groups PM0 to PM5 range from 0.036 to 0.020 . This means that the density of businesses entrances is eighty percent higher on the street segments with access to a monument that in those segments located more than four segments away from a monument.

Conversely, Games-Howell test indicates that the only significant difference between the mean values of business intensity happens between groups PM0 and PM5 ( $p=0.034)$. This suggests that the concentration of businesses in street segments with access to monuments is clearly higher than the average concentration of businesses in street segments more than four street segments apart. Business intensity along those street segments in between does not vary significantly. The next most significant difference occurs between PM0 and PM1 (p $=0.073$ ) - the segments that give access to monuments and the adjacent segments.

\section{Spatial accessibility and proximity to monuments}

This section discusses the analysis of the relation between the location of monuments and their spatial accessibility within the street network, for the case of the historic city of Toledo.

First, the study investigates if the street 
segments providing access to monuments show higher spatial accessibility (normalised integration, $r=2000 \mathrm{~m}$ ) than the rest of the street segments of the historic city. To do so, first, street segments are divided in two groups: those with access to a monument and those without access to any. The two-sample t test confirms that street segments with access to a monument are significantly more integrated than the rest of the segments in the historic city-being the confidence interval $(\gamma=95 \%)$ positive $(0.005,0.055)$. However, the mean integration for a street segment with access to a monument is 0.83 while it is 0.80 for a segment without access to a monument. Therefore, the differences in spatial accessibility are statistically significant but less than four per cent higher for the segments with an entrance to a monument.

However, since this study has already classified the street segments in six groups (PM0 to PM5), depending on their proximity to a monument entrance, statistical analysis can further investigate the relation between spatial accessibility (normalised integration, $r=2000$ $\mathrm{m})$ and the location of monuments (PM).

The analysis studies if an ANOVA model can explain the differences in integration among PM groups. Levene test shows the variances of the six groups are significantly different $(\mathrm{L}=2.992 ; \mathrm{p}=0.011)$, and Welch $(\mathrm{W}$ $=20.92 ; \mathrm{p}<0.05)$ and Brown-Forsythe $(\mathrm{B}-\mathrm{F}=$ $18.98 ; \mathrm{p}<0.05)$ tests show there are significant differences between the integration means of the different PM groups. The predicted normalised integration $(\mathrm{r}=2000 \mathrm{~m})$ values for a street segment belonging to groups PM0 to PM5 are 0.831, 0.836, 0.819, 0.803, and 0.765, respectively.

Games-Howell test indicates that mean integration values are significantly different $(\mathrm{p}<0.05)$ between group PM5 and the other five (groups PM0 to PM4), as well as between PM1 and PM4 $(p=0.017)$. This implies that street segments with access to monuments and those linked to them (PM0 and PM1) are significantly more integrated than those street segments two or three segments away from a monument (PM2, PM3 and PM4). Moreover, the average integration of the latter (PM2, PM3 and PM4) is significantly higher than the average integration of the rest of street segments in the historic city (PM5).

If regrouping the street segments according to the significant differences between the means of PM0 to PM5 revealed in the preceding tests, further statistical analysis shows that mean integration for the new groups formed by PM0 and PM1 (PM0-1); PM2, PM3 and PM4 (PM2-4); and PM5 are 0.834, 0.814, and 0.765 , respectively. Statistics show they are all significantly different between each one of the new groups, although the differences are not so relevant, 2.4 per cent between PM0-1 and PM2-4, and 6.4 per cent between PM2-4 and PM5)

\section{Conclusion}

The main objective of this study has been to understand the distribution of ground floor businesses in heritage cities with high tourism activity. Based on previous research and observations the study has investigated the influence of spatial accessibility and the proximity to monuments in the location of economic activities, in the historic city of Toledo, Spain.

This study confirms there are significant differences in spatial accessibility and proximity to monuments between street segments with and without businesses. Therefore, the analysis suggests that both variables affect the location of ground floor business.

The analysis reveals that, in the historic city of Toledo, integration $(\mathrm{r}=1000 \mathrm{~m}$, and $\mathrm{r}=2000 \mathrm{~m}$ ) is useful to anticipate weather a street might support economic activity or not. The study shows there is a specific probability that a random street segment of the historic city has businesses if its spatial accessibility is greater than a certain value. For example, the probability that a street segment with businesses has normalised integration $(r=2000$ $\mathrm{m})$ above 0.79 is 0.75 ; while the probability that a street segment without businesses has normalised integration $(\mathrm{r}=2000 \mathrm{~m})$ below 0.79 is 0.60 . Other characteristics of an urban tissue might explain the location of businesses better, either alone or in combination with the ones investigated here. Urban morphology 
would benefit from further research on this area since the investigated variables are not enough to understand the distribution of economic activities. The analysis shows weak correlations between business intensity and any of the examined space syntax variables (normalised integration and choice for radii 500,1000 , and 2000 metres, and radius $\mathrm{n}$ ). It does not find an accurate model to explain the location of businesses in the old town based on space syntax variables or the proximity to monuments.

However, considering normalised integration $(\mathrm{r}=2000 \mathrm{~m})$ the study finds significant and remarkable differences in the means of business intensity among the street segments of the different quartiles. Similarly, when the analysis investigates the effect of proximity to monuments, the results show significant and notable differences between the mean business intensity of the street segments with access to a monument and those more than four street segments away.

When studying spatial accessibility, the most integrated twenty-five per cent of the street segments shows a mean business intensity of 0.058 , while the mean of the remaining least integrated seventy-five percent is 0.016 , which is less than one business door in a hundred metres. When analysing the proximity to monuments, the concentration of businesses in street segments with access to monuments is 0.036 while the average concentration of businesses in street segments more than four street segments apart is 0.020 , which is one business door in a hundred metres.

Considering street vitality in relation with business intensity, mean business intensity values (0.016 and 0.020) suggest that the average seventy-five per cent least integrated street segment, as well as the average street segment located more than four segments away from a monument entrance are inactive, according to Gehl's active-to-inactive classification of buildings' ground floors (Gehl, Kaefer, \& Reigstad, 2006).

To better understand the impact of monuments, future research could extend this study in two ways: (1) expanding the street segments classification of PM5 in PM6, PM7, and so on, considering the precise number of segments that separate them from a monument entrance; (2) differentiating monuments in different types - e.g. attending at the number of visitors or users, their actual use, etcetera.

About the location of monuments and spatial accessibility, the study revealed statistically significant differences between the accessibility of the streets segments with access to monuments and the segments without access. Street segments also show significant differences in their integration $(r=2000 \mathrm{~m})$ level, depending on the number of segments away they are from a monument entrance. However, the differences are relatively moderate.

Ongoing research is already applying the same methods to investigate the location of businesses in other Spanish heritage cities to contrast the results obtained for Toledo. Additional studies in different countries and world regions to compare and see how generalizable are the findings presented in this paper.

\section{Acknowledgments}

This research has been funded by the Spanish Ministry of Economy and Competitiveness through the project Tejidos históricos, paisaje urbanos y movilidad. Análisis y propuestas de regeneración de áreas de borde, espacios públicos y ejes viarios (CSO2015-63815-R).

\section{References}

Arnaiz, M., Ruiz-Apilánez, B., \& Ureña, J. M. de. (2013) 'El análisis de la traza mediante Space Syntax. Evolución de la accesibilidad configuracional de las ciudades históricas de Toledo y Alacalá de Henares [Spatial network analysis based on Space Syntax Methodologies. The evolution of integration in the historic cities of Toledo and Alcalá de Henares]', ZARCH Journal of Interdisciplinary Studies in Architecture and Urbanism, 1, 128-140.

Conzen, M. R. G. (1960) 'Alnwick, Northumberland: A Study in Town-Plan Analysis', Transactions and Papers (Institute 
of British Geographers), 27.

Froy, F. (2015) 'Understanding the spatial organisation of economic activities in early 19th century Antwerp', in Proceedings of the 10th International Space Syntax Symposium (London) 49:1-16

Gehl, J., Kaefer, L. J., \& Reigstad, S. (2006) 'Close encounters with buildings', Urban Design International, 11(1), 29-47

Hanson, J. (1989) 'Order and structure in urban design: the plans for the rebuilding of London after the Great Fire of 1666'. Ekistics，334-335(Jan/Feb-Mar/Apr), 2242.

Hillier, B., \& Vaughan, L. (2007) 'The City as One Thing', Progress in Planning, 67(3), 205-230.

Karimi, K. (2000) 'Urban conservation and spatial transformation. Preserving the fragments or maintining the spatial spirit', Urban Design International, 5, 221-231.

Kim, H.-K., \& Sohn, D. W. (2002) 'An analysis of the relationship between land use density of office buildings and urban street configuration', Cities, 19(6), 409-418.

Lee, I. H., \& Kim, Y. O. (2009) 'The Effect of Spatial Configuration and Land Use Pattern on Land Price Formation', in Proceedings of the 7th International Space Syntax Symposium (pp. 1-12) Stockholm.

Min, K. M., Moon, J. M., \& Kim, Y. O. (2007) 'The effect of spatial configuration on land use and land value in Seoul', in Proceedings of the 6th International Space Syntax Symposium. Istambul.

Narvaez, L., Penn, A., \& Griffiths, S. (2013) 'Spatial configuration and bid rent theory: How urban space shapes the urban economy', in Proceedings of the 9th International Space Syntax Symposium 89:1-19.

Ruiz-Apilánez, B., Arnaiz, M., \& Ureña, J. M. de. (2015) 'Beyond Lively Streets', in L. Vaughan (Ed.), Suburban Urbanities (Londres: UCL Press) 130-150

Shen, Y., \& Karimi, K. (2017) 'The economic value of streets: mix-scale spatio-functional interaction and housing price patterns', Applied Geography, 79(January), 187-202.

Troitiño, M. Á., Brandis, D., del Río, I., De la Calle, M., Gutiérrez Puebla, J., Lobo, P., \& Martín, F. (1998) Toledo: problemáticas e implicaciones urbanas en el turismo [Toledo: urban issues and implications for toursim]. Ería, 47, 229-325.

Zárate, M. A. (2007) Estrategias y prácticas turísticas en una ciudad patrimonio de la humanidad, Toledo [Tourist Strategies and Practices in a Wold Heritage city, Toledo]. Geographicalia, 51, 81-106. 